PROCEEDINGS OF THE

AMERICAN MATHEMATICAL SOCIETY

Volume 130, Number 8, Pages 2443-2449

S 0002-9939(02)06306-

Article electronically published on February 4, 2002

\title{
BRANCHED COVERINGS AND NONZERO DEGREE MAPS BETWEEN SEIFERT MANIFOLDS
}

\author{
HONG HUANG
}

(Communicated by Ronald A. Fintushel)

\begin{abstract}
In this note we give a necessary and sufficient condition for the existence of a fiber preserving branched covering between two closed, orientable Seifert manifolds (for sufficiency we need the additional assumption that the genus of the base orbifold of the target manifold $\geq 1$ ). Combining this with two theorems of Rong we get a necessary and sufficient condition for the existence of a nonzero degree map between two such manifolds.
\end{abstract}

\section{InTROdUCTION}

In 7 Yongwu Rong proved:

Theorem. Let $f: M \rightarrow N$ be a nonzero degree map between closed $P^{2}$-irreducible Seifert manifolds of infinite $\pi_{1}$. Then $f \simeq p g \pi$, where $\pi$ is a composition of finitely many vertical pinches, $g$ is a fiber preserving branched covering, and $p$ is a covering. Furthermore, $p$ can be chosen to be the identity map unless $N$ is a Euclidean manifold.

Using this theorem Rong [8] gave a necessary and sufficient condition for the existence of a degree one map between two aspherical, closed, orientable Seifert manifolds (see Theorem 3.2 of [8]), and proved as a corollary: If $M$ is an aspherical, closed, orientable Seifert manifold, then there are only finitely many aspherical Seifert manifolds $N$ such that there exists a degree one map $f: M \rightarrow N$ (see Corollary 4.1 of $[8]$ ).

In this note we try to generalize the results above in 8 to the case of arbitrary degree (but for sufficiency we need the additional condition that the orbifold genus of the target manifold $\geq 1$ ). In Section 1 we give two general facts about branched coverings of Seifert manifolds, which will not be used in the later sections. Then we give a necessary and sufficient condition that there exist a fiber preserving branched covering between two closed, orientable Seifert manifolds (for sufficiency we need the additional assumption that the orbifold genus of the target manifold $\geq 1$ ) (see Theorem 2.2 in Section 2). One of the key ingredients in the proof is a theorem of Husemoller (see Theorem 4 of [4] and Proposition 3.3 of [2]) which guarantees the existence of the branched covering of a closed, orientable surface with nonpositive Euler characteristic with any given branch data with even total branching. As a

Received by the editors October 27, 2000 and, in revised form, February 21, 2001.

2000 Mathematics Subject Classification. Primary 57N10; Secondary 57M12, 55M25.

Key words and phrases. Seifert manifold, branched covering, nonzero degree map. 
consequence, we get an elementary proof of the naturality of the Euler numbers of Seifert manifolds [6]. Combining Theorem 2.2 with Rong's theorems above we get a necessary and sufficient condition that there exist a nonzero degree map between two such manifolds (see Theorem 3.0 in Section 3). As a consequence, we show that for a given aspherical, closed, oriented Seifert manifold $M$ and a nonzero integer $d$, there are only finitely many such Seifert manifolds $N$ such that there is a degree $d$ map $f: M \rightarrow N$ (see Corollary 3.1).

\section{Two GENERAL FACTS ABOUt BRANCHED COVERINGS OF SEIFERT MANIFOLDS}

In this section we generalize two well-known facts about coverings of Seifert manifolds to the case of branched coverings, which will not be used in the later sections.

Proposition 1.0. A finite sheeted branched covering space of a Seifert manifold branched over fibers admits a Seifert fibration.

The proof is easy and omitted.

Remark. If we remove the condition "branched over fibers", then the result is obviously not true.

Conversely, we have

Proposition 1.1. Let $S$ be a compact, connected, irreducible, orientable 3-manifold. Suppose that some finite sheeted branched covering space $S^{\prime}$ of $S$ admits a Seifert fibration such that the inverse image of the downstairs branch set consists of some fibers. Then $S$ itself admits a Seifert fibration such that the downstairs branch set consists of some fibers.

Proof. Suppose we have a finite sheeted branched covering $p: S^{\prime} \rightarrow S$, where $S^{\prime}$ is a Seifert manifold and the inverse image of the downstairs branch set consists of some fibers. Let $p \mid: S^{\prime}-p^{-1}\left(N\left(B_{p}\right)\right) \rightarrow S-N\left(B_{p}\right)$ be the corresponding unbranched covering, where $B_{p}$ is the downstairs branch set and $N\left(B_{p}\right)$ is an open regular neighborhood of $B_{p}$ such that $p^{-1}\left(N\left(B_{p}\right)\right)$ is fibered. By [5, Theorem 6.3] and the positive solution of the Seifert fibered space conjecture by Gabai, Casson and Jungreis [1], 3], $S-N\left(B_{p}\right)$ admits a Seifert fibration, which can extend to $N\left(B_{p}\right)$ such that $B_{p}$ consists of some fibers as is easily seen.

Note that for any nonzero degree map $f: M \rightarrow N$ between two aspherical, closed Seifert manifolds, we have $f \simeq f_{1} p$, where $p: M \rightarrow M_{1}$ is a fiber preserving branched covering (in fact $p$ is the natural projection from $M$ to some $M / Z_{n}$, where $Z_{n}$ is in the $S^{1}$ action on $\left.M\right)$, and $f_{1 *}(h)=h$, where $h$ represents regular fibers of both $M, N$. So we shall first consider nonzero degree map $f: M \rightarrow N$ of fiber degree 1 in the next section.

\section{FIBER PRESERVING BRANCHED COVERINGS BETWEEN SEIFERT MANIFOLDS}

From now on, "Seifert manifold" will always mean an oriented closed connected 3 -manifold admitting a fixed point free action of $S^{1}$. As is well known, such a manifold is equivariantly classified by its "Seifert invariants" [10]. We shall use unnormalized Seifert invariants (see [6]). The unnormalized Seifert invariant of a Seifert 
manifold $M$ is denoted by $I(M)=\left\{g ;\left(\alpha_{1}, \beta_{1}\right), \ldots,\left(\alpha_{m}, \beta_{m}\right)\right\}$ or $\left\{g ; r_{1}, \ldots, r_{m}\right\}$ where $g$ is the orbifold genus of $M,\left(\alpha_{i}, \beta_{i}\right)\left(\alpha_{i}, \beta_{i}\right.$ coprime and $\left.\alpha_{i} \geq 1\right)$ are the Seifert invariants of the maybe-singular fibers, and $r_{i}=\beta_{i} / \alpha_{i}$.

The unnormalized Seifert invariant of a Seifert manifold $M$ is not unique. We can transform it while not changing the isomorphic type of the Seifert fibration of $M$ by the following moves which we call equivalent moves (see [6], [8]):

(1) permute the $r_{i}$.

(2) add 0 to or delete 0 from the list $r_{1}, \ldots, r_{n}$.

(3) replace $r_{i}, r_{j}$ by $r_{i}+1, r_{j}-1$.

As preparation, let's see when there exists a $k$-fold fiber preserving branched covering of fiber degree 1 between two Seifert fibered solid tori $V_{1}$ and $V_{2}$ such that upstairs and downstairs branch sets are their central fibers.

Let the Seifert invariant of $V_{2}$ be $\left(\alpha_{2}, \beta_{2}\right)$ under a (cross section, fiber) basis $\left(c_{2}, h_{2}\right)$, i.e. the meridian $\mu_{2}$ of $V_{2}$ can be represented by $c_{2}^{\alpha_{2}} h_{2}^{\beta_{2}}$. If such a branched covering $f: V_{1} \rightarrow V_{2}$ exists, then $c_{2}$ lifts to a cross section $c_{1}$ of the fibration of $V_{2}$ restricted to $\partial V_{2}$ for fiber degree one. Thus we have $\left.f\right|_{*}\left(c_{1}\right)=c_{2}^{k}$, where $f \mid$ is the restriction of $f$ to $\partial V_{1}$.

By the definition of branched covering we can write $\left.f\right|_{*}\left(\mu_{1}\right)=\mu_{2}^{s}$, where $s$ is some nonzero integer. So $\left.f\right|_{*}\left(c_{1}^{\alpha_{1}} h_{1}^{\beta_{1}}\right)=\left(c_{2}^{\alpha_{2}} h_{2}^{\beta_{2}}\right)^{s}$, where we set the Seifert invariant of $V_{1}$ under the (section, fiber) basis $\left(c_{1}, h_{1}\right)$ to be $\left(\alpha_{1}, \beta_{1}\right)$. Thus $c_{2}^{k \alpha_{1}} h_{2}^{\beta_{1}}=c_{2}^{s \alpha_{2}} h_{2}^{s \beta_{2}}$, i.e. $k \alpha_{1}=s \alpha_{2}, \beta_{1}=s \beta_{2}$. So $\beta_{1} / \alpha_{1}=k \beta_{2} / \alpha_{2}$.

Conversely, if $V_{i}$ has Seifert invariant $\left(\alpha_{i}, \beta_{i}\right)$, and $\alpha_{i}, \beta_{i}(i=1,2)$, and $k$ satisfy the relation above, then clearly there exists a branched covering $V_{1} \rightarrow V_{2}$ satisfying the given condition (by a direct construction; cf. [10]).

Definition 2.0. Let $R=\left\{\beta_{1} / \alpha_{1}, \ldots, \beta_{s} / \alpha_{s}\right\}, R^{\prime}=\left\{\beta_{1}^{\prime} / \alpha_{1}^{\prime}, \ldots, \beta_{k}^{\prime} / \alpha_{k}^{\prime}\right\}$ be two unordered sets of rational numbers (repetitions allowed). Let $d$ be a positive integer. We say $R \geq_{d} R^{\prime}$ if $R$ can be split into a "disjoint" union $R=R_{1} \cup \ldots \cup R_{k}$ such that for any $i(1 \leq i \leq k)$ and any $\beta_{j} / \alpha_{j} \in R_{i}, \beta_{j} / \alpha_{j}=a_{j} \beta_{i}^{\prime} / \alpha_{i}^{\prime}$ (where $a_{j}$ is some positive integer), and $\sum a_{j}=d$, where the summation is over all $j$ such that $\beta_{j} / \alpha_{j} \in R_{i}$.

Lemma 2.1. Let $M, N$ be closed oriented Seifert manifolds. Suppose $I(N)=$ $\left\{g^{\prime} ; R^{\prime}\right\}$, where $g^{\prime} \geq 1$ and $R^{\prime}=\left\{\beta_{1}^{\prime} / \alpha_{1}^{\prime}, \ldots, \beta_{n}^{\prime} / \alpha_{n}^{\prime}\right\}(n \geq 1)$. Then there is a $d$-fold fiber preserving branched covering $M \rightarrow N$ with fiber degree one if and only if $M$ has Seifert invariant $\{g ; R\}$, where $R \geq_{d} R^{\prime}$ and $n d-|R| \leq 2 d\left(1-g^{\prime}\right)-2(1-g)$. Here $|R|$ is the number of elements in the multiset $R$ (counted with multiplicity).

Proof. Suppose there is such a branched covering $f: M \rightarrow N$. Recall how we get the Seifert invariant of $N$ as in [6]. Let $O_{1}, \ldots, O_{n}$ be a nonempty collection of fibers in $N$, including all singular fibers. Let $T_{1}, \ldots, T_{n}$ be disjoint Seifert fibered tubular neighborhoods of $O_{1}, \ldots, O_{n}$ and $N_{0}=N-i n t\left(T_{1} \cup \ldots \cup T_{n}\right)$. Take the section $c_{2}$ of the bundle $N_{0} \rightarrow N_{0} / S^{1}$ which leads to the Seifert invariant $\left\{g^{\prime} ; R^{\prime}\right\}$ of $N$. Let $M_{0}=$ $M-f^{-1}\left(\operatorname{int}\left(T_{1} \cup \ldots \cup T_{n}\right)\right)$. Then $c_{2}$ lifts to a section $c_{1}$ of the bundle $M_{0} \rightarrow M_{0} / S^{1}$ for fiber degree one. Let $\{g ; R\}$ be the Seifert invariant of $M$ under the (section, fiber) frame $\left(c_{1}, h_{1}\right)$, where $h_{1}$ stands for a regular fiber of $M$. Considering $T_{i}$ and its inverse image under $f$ we get the relation $R \geq_{d} R^{\prime}$ by the local computation before Definition 2.0. The inequality $n d-|R| \leq 2 d\left(1-g^{\prime}\right)-2(1-g)$ follows from the Riemann-Hurwitz formula since $n d-|R|$ is a part of the total branching of the induced branched covering $\bar{f}: M / S^{1} \rightarrow N / S^{1}$. 
Conversely suppose the Seifert invariants of $M, N$ satisfy the relation in the lemma we'll go to construct a branched covering $f: M \rightarrow N$ as required. Let

$$
k=2 d\left(1-g^{\prime}\right)-2(1-g)-(n d-|R|) .
$$

Let $\mathcal{D}=\left\{A_{1}, \ldots, A_{n}, A_{n+1}, \ldots, A_{n+k}\right\}$, where $A_{i}(i=1, \ldots, n)$ are the partitions of $d$ given by the relation $R \geq{ }_{d} R^{\prime}$ (i.e. each $A_{i}$ consists of the $a_{j}$ 's (depending on $i$ ) in Definition 2.0), $A_{n+j}=[2,1, \ldots, 1](d-2$ times 1$)$ for $j=1, \ldots, k$. By Theorem 4 of [4] or Proposition 3.3 of [2] there is a $d$-fold branched covering $\bar{f}: F_{g} \rightarrow F_{g^{\prime}}$ with branch data $\mathcal{D}$, where $F_{g}$ is the closed, orientable surface of genus $g$. Based on this and the local constructions (as what we have done in the preparation before Definition 2.0) decided by the relation $R \geq_{d} R^{\prime}$, a branched covering $M \rightarrow N$ as required can be constructed: We have the induced covering between surfaces with boundary $\bar{f} \mid: F_{g}-\bar{f}^{-1}\left(\operatorname{int}\left(D_{1} \cup \ldots \cup D_{n+k}\right)\right) \rightarrow F_{g^{\prime}}-\operatorname{int}\left(D_{1} \cup \ldots \cup D_{n+k}\right)$. Then clearly

$$
\begin{aligned}
\bar{f} \mid \times i d:\left(F_{g}-\bar{f}^{-1}\left(\operatorname { i n t } \left(D_{1} \cup \ldots \cup\right.\right.\right. & \left.\left.\left.D_{n+k}\right)\right)\right) \\
& \times S^{1} \rightarrow\left(F_{g^{\prime}}-\operatorname{int}\left(D_{1} \cup \ldots \cup D_{n+k}\right)\right) \times S^{1}
\end{aligned}
$$

extends to a $d$-fold fiber preserving branched covering $f: M \rightarrow N$ with fiber degree one.

Remark. In the proof of the necessity of the lemma above we do not need the condition $g^{\prime} \geq 1$. But for sufficiency we do need $g^{\prime} \geq 1$ as shown in the following example.

Example. Let $I(M)=\{0 ; R\}=\{0 ; 2 / 13,2 / 13,4 / 17,4 / 17,9 / 19,3 / 19\}, I(N)=$ $\left\{0 ; R^{\prime}\right\}=\{0 ; 1 / 13,2 / 17,3 / 19\}$. Then $R \geq_{4} R^{\prime}$ and $n d-|R|=6=2 d\left(1-g^{\prime}\right)-$ $2(1-g)$. However there is no 4 -fold fiber preserving branched covering $M \rightarrow N$ with fiber degree one, for $\{[2,2],[2,2],[3,1]\}$ cannot arise as the branch data of a connected branched covering of $S^{2}$ (see Proposition 5.3 of [2]).

In the case of arbitrary fiber degree we have the following:

Theorem 2.2. Let $M, N$ be closed, oriented Seifert manifolds with Seifert invariants

$$
\begin{aligned}
I(M) & =\{g ; R\}=\left\{g ; \beta_{1} / \alpha_{1}, \ldots, \beta_{m} / \alpha_{m}\right\}, \\
I(N) & =\left\{g^{\prime} ; R^{\prime}\right\}=\left\{g^{\prime} ; \beta_{1}^{\prime} / \alpha_{1}^{\prime}, \ldots, \beta_{n}^{\prime} / \alpha_{n}^{\prime}\right\},
\end{aligned}
$$

where $g^{\prime} \geq 1$. Let $d, d_{1}$ be positive integers and $d_{1} \mid d$. Then there is a $d$-fold fiber preserving branched covering $M \rightarrow N$ with fiber degree $d_{1}$ if and only if $d_{1} R:=$ $\left\{d_{1} \beta_{1} / \alpha_{1}, \ldots, d_{1} \beta_{m} / \alpha_{m}\right\}$ can be equivalently moved to a set $\bar{R} \geq_{d_{2}} R^{\prime}$ (where $d_{2}=$ $\left.d / d_{1}\right)$ and $n d_{2}-|\bar{R}| \leq 2 d_{2}\left(1-g^{\prime}\right)-2(1-g)$.

Proof. First we prove necessity. If there exists such a branched covering $f: M \rightarrow N$, then $f$ factors through $M /\left(Z / d_{1}\right)$ (where $Z / d_{1}$ is inside the $S^{1}$ action on $M$ ). By a fact due to Seifert (see [10] or Lemma 1.3 of [6] $) M /\left(Z / d_{1}\right)$ has Seifert invariant $\left\{g ; d_{1} \beta_{1} / \alpha_{1}, \ldots, d_{1} \beta_{m} / \alpha_{m}\right\}$. Now the necessity clearly follows from the lemma above.

Sufficiency can be proved by a direct construction as in the lemma above.

Remark. Again in the proof of the necessity $g^{\prime} \geq 1$ is not used. 
As an application we have the following.

Corollary 2.3 ([6]). Let $M, N$ be aspherical, closed, oriented Seifert manifolds. Suppose there is a degree $d(d \neq 0)$ fiber preserving map $f: M \rightarrow N$ with fiber degree $d_{1}$. Then $e(M)=\left(d_{2} / d_{1}\right) e(N)$, where $e(M)$ (resp. $\left.e(N)\right)$ is the Euler number of the Seifert fibration of $M(\operatorname{resp} . N)$, and $d_{2}=d / d_{1}$.

Proof. By the theorem of Rong cited at the beginning of this note, $f \simeq g \pi$, where $\pi: M \rightarrow \hat{M}$ is a composition of finitely many of vertical pinches, and $g: \hat{M} \rightarrow N$ is a fiber preserving branched covering. By Corollary 3.3 of $8 \quad e(M)=e(\hat{M})$. We can write $g=g_{2} g_{1}$, where $g_{1}: \hat{M} \rightarrow \bar{M}:=\hat{M} /\left(Z / d_{1}\right)$ is the natural projection, and $g_{2}: \bar{M} \rightarrow N$ is a $d_{2}$-fold fiber preserving branched covering of fiber degree one. By the fact due to Seifert cited above, $e(\hat{M})=\left(1 / d_{1}\right) e(\bar{M})$. By Theorem 2.2 and Definition 2.0, $e(\bar{M})=d_{2} e(N)$. So $e(M)=\left(d_{2} / d_{1}\right) e(N)$.

Remark. The proof of the fact above sketched in [6] used more algebraic topology, while our proof here is quite elementary.

Corollary 2.4 ([8]). Let $M, N$ be closed, oriented Seifert manifolds, where $\chi\left(O_{N}\right)$ $\leq 0$. Suppose there is a d-fold fiber preserving branched covering $f: M \rightarrow N$ with orbifold degree $d_{2}$. Then $\left|\chi\left(O_{M}\right)\right| \geq d_{2}\left|\chi\left(O_{N}\right)\right|$, where $O_{M}\left(\right.$ resp. $\left.O_{N}\right)$ is the base orbifold of $M($ resp. $N)$ and $\chi\left(O_{M}\right)\left(\right.$ resp. $\left.\chi\left(O_{N}\right)\right)$ is its Euler characteristic. The equality holds if and only if $f$ is a covering.

Proof. It follows from a direct computation using the Theorem.

Let $\bar{M}=M /\left(Z / d_{1}\right)$, where $d_{1}=d / d_{2}$. We have

$$
\begin{aligned}
& -\chi\left(O_{\bar{M}}\right)+d_{2} \chi\left(O_{N}\right) \\
= & -\chi\left(\left|O_{\bar{M}}\right|\right)+\sum_{i=1}^{|\bar{R}|}\left(1-1 / \bar{\alpha}_{i}\right)+d_{2} \chi\left(\left|O_{N}\right|\right)-d_{2} \sum_{j=1}^{n}\left(1-1 / \alpha_{j}^{\prime}\right) \\
= & d_{2} \chi\left(\left|O_{N}\right|\right)-\chi\left(\left|O_{\bar{M}}\right|\right)-\left(n d_{2}-|\bar{R}|\right)+\sum_{j=1}^{n}\left(d_{2} / \alpha_{j}^{\prime}-\sum_{\bar{\beta}_{i} / \bar{\alpha}_{i} \in \bar{R}_{j}} 1 / \bar{\alpha}_{i}\right) \\
\geq & d_{2} \chi\left(\left|O_{N}\right|\right)-\chi\left(\left|O_{\bar{M}}\right|\right)-\left(n d_{2}-|\bar{R}|\right) \quad\left(\text { using the relation } \bar{R} \geq_{d_{2}} R^{\prime}\right) \\
\geq & 0 .
\end{aligned}
$$

The last two equalities hold if and only if $\bar{M} \rightarrow N$ is a covering.

On the other hand, $-\chi\left(O_{M}\right) \geq-\chi\left(O_{\bar{M}}\right)$ by the fact due to Seifert cited above; the equality holds if and only if $\bar{f}: M \rightarrow \bar{M}$ is a covering.

Combining these two things the proof is complete.

Remark. It is indicated in [8] that the inequality in Corollary 2.4 can be proved by a covering space argument. This in turn depends on the fact that every good, compact 2-dimensional orbifold without boundary is finitely covered by a manifold (see for example [9]). But the proof of this fact is not very easy. We also note that the inequality has independent meaning, i.e. it can be applied directly to 2-orbifold branched coverings.

We note that however the condition " $n d-|R| \leq 2 d\left(1-g^{\prime}\right)-2(1-g)$ " in Lemma 2.1 cannot be replaced by $\left|\chi\left(O_{M}\right)\right| \geq d\left|\chi\left(O_{N}\right)\right|$ as shown in the following example.

Example. Let $I(M)=\{5 ; R\}=\{5 ; 2 / 5,3 / 5,2 / 7,3 / 7,2 / 11,3 / 11\}, I(N)=\left\{1 ; R^{\prime}\right\}$ $=\{1 ; 1 / 5,1 / 7,1 / 11\}, d=5$. Then $R \geq_{5} R^{\prime}$ and $\left|\chi\left(O_{M}\right)\right| \geq 5\left|\chi\left(O_{N}\right)\right|$. But it is easy to see that $R$ cannot be equivalently moved to a set $R_{1}$ such that $R_{1} \geq_{5} R^{\prime}$ and $15-\left|R_{1}\right| \leq 10(1-1)-2(1-5)=8$. 
Similarly using Theorem 4 of [4] (or Proposition 3.3 of [2]) we have the following.

Proposition 2.5. Let $N$ be a closed, orientable Seifert manifold with Seifert invatiant $I(N)=\left\{g^{\prime} ;\left(\alpha_{1}^{\prime}, \beta_{1}^{\prime}\right), \ldots,\left(\alpha_{n}^{\prime}, \beta_{n}^{\prime}\right)\right\}$, where $g^{\prime} \geq 1$. Suppose $d, d_{2}$ are two positive integers and $d_{2} \mid d$. Let $\mathcal{D}=\left\{A_{1}, \ldots, A_{n}, A_{n+1}, \ldots, A_{n+k}\right\}$ be a collection of partitions of $d_{2}$ (with repetitions allowed). Then there is a closed, oriented Seifert manifold $M$ and a d-fold fiber preserving branched covering $M \rightarrow N$ with orbifold degree $d_{2}$ and orbifold branch data $\mathcal{D}$ (the corresponding branch set in $N / S^{1}$ is the $n$ cone points and $k$ regular points) if and only if the total branching $v(\mathcal{D}):=(n+k) d_{2}-\sum_{l=1}^{n+k}\left|A_{l}\right|$ is even, where $\left|A_{l}\right|$ denotes the number of components of $A_{l}$ (abusing notation).

Remark. A cone point of the orbifold $O_{N}$ of $N$ may be a downstairs branch point of the induced map $\bar{f}: O_{M} \rightarrow O_{N}$ while the corresponding singular fiber is not in the downstairs branch set of $f: M \rightarrow N$, and vice versa.

\section{Applications to nonzero Degree maps BETWEEN SEIFERT MANIFOLDS}

Now we can generalize Theorem 3.2 of 8 to the case of arbitrary degree (but for sufficiency we need the additional assumption that the orbifold genus of the target manifold $\geq 1$ ).

Theorem 3.0. Let $M, N$ be aspherical, closed, oriented Seifert manifolds with Seifert invariants $I(M)=\{g ; R\}, I(N)=\left\{g^{\prime} ; R^{\prime}\right\}$, where $g^{\prime} \geq 1$. Let $d$ be a positive integer. Suppose $N$ admits no Euclidean geometry. Then there is a map $M \rightarrow N$ with degree $d$ if and only if there is a set $\{\hat{g} ; \hat{R}\}$ such that $g \geq \hat{g}$ and $R \geq \hat{R}$ (which is defined in $\left[\underline{8}\right.$ ), and $\{\hat{g} ; \hat{R}\}$ and $\left\{g^{\prime} ; R^{\prime}\right\}$ satisfy the relation in Theorem 2.2 (satisfied by $\{g ; R\}$ and $\left\{g^{\prime} ; R^{\prime}\right\}$ ) for some integer $d_{1} \mid d$.

Proof. First we prove necessity. If there is a map $f: M \rightarrow N$ of degree $d$, then by the theorem of Rong cited at the beginning of this note we have $f \simeq g \pi$, where $\pi: M \rightarrow \hat{M}$ is a composition of finitely many vertical pinches, and $g$ is a fiber preserving branched covering. By Theorem 3.2 of $8, \hat{M}$ has Seifert invariant $\{\hat{g} ; \hat{R}\}$ which satisfies $g \geq \hat{g}$ and $R \geq \hat{R}$. Now the necessity follows from Theorem 2.2 .

Sufficiency follows immediately from Theorem 3.2 of [8] and Theorem 2.2.

Now we have the following generalization of Corollary 4.1 of [8].

Corollary 3.1. Let $M$ be an aspherical, closed, oriented Seifert manifold. Then given a nonzero integer $d$, there are only finitely many aspherical, closed, oriented Seifert manifolds $N$ such that there is a map $f: M \rightarrow N$ with degree $d$.

Proof. We may assume $d>0$. Suppose $N$ is an aspherical, closed, oriented Seifert manifold such that there is a map $f: M \rightarrow N$ with degree $d$. Note that there are only 10 closed Euclidean 3-manifolds, so we can assume that $N$ admits no Euclidean geometry. Then the Seifert invariants of $M$ and $N$ have the relation as described in Theorem 3.0. By Corollary 4.1 of [8] there are only finitely many aspherical, closed, oriented Seifert manifolds $\hat{M}$ such that there is a degree one map $M \rightarrow \hat{M}$. So we need only show that given an aspherical, closed, oriented Seifert manifold $\hat{M}$, there are only finitely many aspherical, closed, oriented Seifert manifolds $N$ such that there is a $d$-fold fiber preserving branched covering $\hat{M} \rightarrow N$. By Corollary $2.4-\chi\left(O_{\hat{M}}\right) \geq-d_{2} \chi\left(O_{N}\right)$, where $d_{2}$ is the orbifold degree. Let $\bar{M}=\hat{M} /\left(Z / d_{1}\right)$, 
where $d_{1}=d / d_{2}$. From Theorem 2.2 and Definition 2.0 we have $\bar{\alpha}_{j} \leq \alpha_{i}^{\prime} \leq d_{2} \bar{\alpha}_{j}$ for any $i$, where $\bar{\beta}_{j} / \bar{\alpha}_{j} \in \bar{R}_{i}$. There are only finitely many 2-orbifolds $O_{N}$ which satisfy these two conditions. Also $e(N)=\left(d_{1} / d_{2}\right) e(M)$. So there are only finitely many such $N$.

Corollary 3.2. Let $M, N$ be aspherical, closed, oriented Seifert manifolds with given Seifert invariants, where the orbifold genus of $N \geq 1$. Then given a nonzero integer $d$ there is an algorithm to decide whether there is a map $f: M \rightarrow N$ of degree $d$. Furthermore, if $e(N) \neq 0$ and $\chi\left(O_{N}\right) \neq 0$ the set $\{d$ : there is a map $f$ : $M \rightarrow N$ of degree $d\}$ is finite and can be decided.

Proof. For simplicity we assume $e(N) \neq 0$ and $\chi\left(O_{N}\right) \neq 0$. If there is a map $f$ : $M \rightarrow N$ of degree $d$, say, then $e(M) \neq 0$ and $|d| \leq|e(N)| \chi\left(O_{M}\right)^{2} /\left(|e(M)| \chi\left(O_{N}\right)^{2}\right)$ by Corollaries 2.3 and 2.4. We can find the finitely many Seifert manifolds $\hat{M}$ such that there is a degree one map $M \rightarrow \hat{M}$ by the proof of Corollary 4.1 of [8]. For each $\hat{M}$ let $\bar{M}=\hat{M} /\left(Z / d_{1}\right)$ (where $d_{1}$ is the divisor of $d$ such that $e(M)=\left(d_{2} / d_{1}\right) e(N)$ and $\left|\chi\left(O_{M}\right)\right| \geq d_{2}\left|\chi\left(O_{N}\right)\right|$ (where $\left.d_{2}=d / d_{1}\right)$ ). By Lemma 2.1 and the finiteness of the number of partitions of $d_{2}$ we can decide whether there is a $d_{2}$-fold fiber preserving branched covering $\bar{M} \rightarrow N$ of fiber degree one.

\section{ACKNOWLEDGEMENTS}

I am very grateful to Professor Shicheng Wang for introducing me to this field. I would also like to thank the referee for his helpful comments.

\section{REFERENCES}

[1] A. Casson, D. Jungreis, Convergence groups and Seifert fibered 3-manifolds, Invent. Math. 118 (1994), 441-456. MR 96f:57011

[2] A. L. Edmonds, R. S. Kulkarni and R. E. Stong, Realizability of branched coverings of surfaces, Trans. Amer. Math. Soc. 282 (1984), No. 2, 773-790. MR 85k:57005

[3] D. Gabai, Convergence groups are Fuchisian groups. Ann. of Math. 136 (1992), 447-510. MR 93m:20065

[4] D. Husemoller, Ramified coverings of Riemann surfaces, Duke Math. J. 29 (1962), 167-174. MR 25:188

[5] W. Jaco, P. Shalen, Seifert Fibered Spaces in 3-manifolds, Memoirs of Amer. Math. Soc. 21 (1979) (no. 220). MR 81c:57010

[6] W. D. Neumann, F. Raymond, Seifert manifolds, plumbing, $\mu$-invariant and orientation reversing maps, Lecture Notes in Mathematics 664, 162-195. MR 80e:57008

[7] Y. Rong, Maps between Seifert fibered spaces of infinite $\pi_{1}$, Pacific J. Math. 160 (1993), No. 1, 143-154. MR 94e:55026

[8] Y. Rong, Degree one maps of Seifert manifolds and a note on Seifert volume, Topol. Appl. 64 (1995), 191-200. MR 96c:57034

[9] P. Scott, The geometries of 3-manifolds, Bull. London Math. Soc. 15 (1983), 401-487. MR 84m:57009

[10] H. Seifert, Topologie dreidimensionaler gefaserter Raume, Acta Math. 60 (1933), 147-238.

School of Mathematical Sciences, Peking University, Beijing 100871, People's RePUBLIC OF CHINA

Current address: Nankai Institute of Mathematics, Nankai University, Tianjin 300071, People's Republic of China

E-mail address: hhuang01@263.net 